\title{
On the Extension of $\mathbb{L}^{2}$ Holomorphic Functions II
}

\author{
By \\ Takeo OHSAWA*
}

\section{Introduction}

Let $\left(X, d s^{2}\right)$ be a complete Hermitian manifold of dimension $n$, and let $\varphi$ be a real-valued $C^{\infty}$ function on $X$. By the theory of $\mathbb{L}$. Hörmander $[\mathrm{H}]$, a $\bar{\partial}$ closed form $u$ on $X$ is $\bar{\partial}$-exact if it satisfies the estimate

$$
\left|(u, v)_{\varphi}\right| \leq C_{u}\left(\|\bar{\partial} v\|_{\varphi}+\left\|\bar{\partial}{ }_{\varphi}^{*} v\right\|_{\varphi}\right)
$$

for any compactly supported $C^{\infty}$ form $v$, where $C_{u}$ is a number independent of $v$, and in many cases the estimate is true for $C_{u}=$ const. $\|u\|_{\varphi}$. In our previous work [O-T], we have established a new $L^{2}$-inequality involving the $\bar{\partial}$ operator, in which the estimation for $C_{u}$ is more elaborate. As a consequence, it enabled us to prove the following.

Theorem. Let $D$ be a bounded pseudoconvex domain in $\mathbb{C}^{n}$ and let $H \subset \mathbb{C}^{n}$ be a complex hyperplane. Then, every $L^{2}$ holomorphic function on $D \cap H$ has an $L^{2}$ holomorphic extension to $D$.

The purpose of the present paper is to formulate and prove a generalized $L^{2}$ extension theorem from higher codimensional submanifolds which includes our previous result as a special case, by using our new $L^{2}$ inequality.

Our main result is as follows.

Theorem. Let $X$ be a Stein manifold of dimension $n, Y \subset X$ a closed complex submanifold of codimension $m$, and $(E, h)$ a Nakano-semipositive vector bundle over $X$. Let $\varphi$ be any plurisubharmonic function on $X$ and let $s_{1}, \cdots, s_{m}$ be holomorphic functions on $X$ vanishing on $Y$. Then, given a holomorphic E-valued $(n-m)$-form $g$ on $Y$ with

Received August 1, 1987.

* Research Institute for Mathematical Sciences, Kyoto University, Kyoto 606, Japan. 


$$
\left|\int_{Y} e^{-\varphi} h(g) \wedge \bar{g}\right|<\infty
$$

there exists for any $\varepsilon>0$, a holomorphic E-valued $n$-form $G_{\varepsilon}$ on $X$ which coincides with $g \wedge d s_{1} \wedge \cdots \wedge d s_{m}$ on $Y$ and satisfies

$$
\left|\int_{X} e^{-\varphi}\left(1+|s|^{2}\right)^{-m-\varepsilon} h\left(G_{\varepsilon}\right) \wedge \bar{G}_{\varepsilon}\right| \leq \varepsilon^{-1} C_{m}\left|\int_{Y} e^{-\varphi} h(g) \wedge \bar{g}\right|
$$

where $|s|^{2}=\sum_{i=1}^{m}\left|s_{i}\right|^{2}$ and $C_{m}$ is a positive number which depends only on $m$.

To see that one cannot drop $\varepsilon$ in (1), it suffices to consider the case $X=\boldsymbol{C}$, $Y=\{0\}, \varphi=0$ and $g=1$.

Among a few direct consequences of Theorem, the following two observations might be of interest.

Corollary 1. Let $X$ be a weakly 1-complete manifold of dimension $n$ which admits a positive line bundle, let $s$ be a holomorphic function on $X$ such that $d s \neq 0$ on $Y:=s^{-1}(0)$, and let $(E, h)$ be a Nakano semipositive vector bundle over $X$. Then the restriction map

$$
\Gamma\left(X, \mathcal{O}_{X}\left(K_{X} \otimes E\right)\right) \rightarrow \Gamma\left(Y, \mathcal{O}_{Y}\left(K_{X} \otimes E\right)\right)
$$

is surjective.

Corollary 2. Let $Y$ be a pure dimensional closed complex submanifold of $C^{N}$, let $\Omega$ be a bounded domain of holomorphy, and $\varphi$ a plurisubharmonic function on $\Omega$. Then, for any holomorphic function $f$ on $\Omega \cap Y$ with

$$
\int_{\Omega \cap Y} e^{-\varphi}|f|^{2} d V_{Y}<\infty
$$

there exists a holomorphic extension $F$ to $\Omega$ such that

$$
\int_{\Omega} e^{-\varphi}|F|^{2} d V \leq A \int_{\Omega \cap Y} e^{-\varphi}|f|^{2} d V_{Y}
$$

Here $A$ depends on $Y$ and $\sup \{\|z\| ; z \in \Omega\}$, but does not depend on $f$.

In $\S 1$ we improve the estimates shown in [O-T] and [O-2], so that one can dispense with auxiliary complete Kähler metrics which we needed before. We shall prove Theorem in $\S 2$ by solving $\bar{\partial}$-equations on a family of strongly pseudoconvex domains and taking a limit of solutions. In case $m=1$, it amounts to solve the equation

$$
\bar{\partial} u=2 \pi i g \wedge[Y]
$$


with an appropriate $L^{2}$ estimate, where $[Y]$ denotes the $(1,1)$-current associated to $Y$. For $m \geq 2$ the limit equation is hard to describe in the framework of distributions, and it might be interesting to know its legitimate description.

\section{$\S 1 . \quad$ Notations and Preliminaries}

Let $\left(X, d s^{2}\right)$ be a Kähler manifold of dimension $n,(E, h)$ a Hermitian vector bundle over $X$ and $\varphi$ a $C^{\infty}$ real valued function on $X$. We shall use the following notations.

$$
\begin{aligned}
C^{p, q}(X, E)= & \left\{C^{\infty} E \text {-valued }(p, q) \text {-forms on } X\right\} \\
C_{0}^{p, q}(X, E)= & \left\{f \in C^{p, q}(X, E) ; \operatorname{supp} f \subset X\right\} \\
L_{\varphi}^{p, q}(X, E)= & \{\text { measurable } E \text {-valued }(p, q) \text {-forms } f \text { on } X \text { satisfying } \\
& \left.\int_{X} e^{-\varphi}|f|^{2} d V_{X}<\infty\right\},
\end{aligned}
$$

where $|f|$ denotes the length of $f$ and $d V_{X}$ denotes the volume form.

We denote by $\bar{\partial}: L_{\varphi}^{p, q}(X, E) \rightarrow L_{\varphi}^{p, q+1}(X, E)$ the complex exterior derivative of type $(0,1)$ defined on

$$
\operatorname{Dom} \bar{\partial}:=\left\{f \in L_{\varphi}^{p, q}(X, E) ; \bar{\partial} f \in L_{\varphi}^{p, q+1}(X, E)\right\} .
$$

The adjoint of $\bar{\partial}$ will be denoted by $\bar{\partial}_{\varphi}^{*}$.

Let $(f, g)_{\varphi}$ be the inner product of $f, g \in L_{\varphi}^{p, q}(X, E)$ associated to the norm

$$
\|f\|_{\varphi}:=\left(\int_{X} e^{-\varphi}|f|^{2} d V_{X}\right)^{1 / 2}
$$

For a $(p, q)$-form $f$ on $X$ we denote by $e(f)$ the left multiplication by $f$ in the exterior algebra of differential forms on $X$. The (pointwise) adjoint of $e(f)$ is denoted by $e(f)^{*}$. We shall denote by $\omega$ the fundamental form of $d s^{2}$, and put $\Lambda=e(\omega)^{*}$. The curvature form of $h$ will be denoted by $\Theta=\sum_{\alpha, \beta} \Theta_{\alpha \bar{\beta} \nu}^{k} d z^{\alpha} \wedge d \bar{z}^{\beta}$. The left multiplication by $\Theta$ to $E$-valued forms is well-defined and denoted by $e(\Theta) . \quad(E, h)$ is said to be Nakano semipositive if the Hermitian form $\sum_{\alpha, \beta, \nu, \mu}\left(\sum_{\kappa}\right.$ $\left.\Theta_{\alpha \bar{\beta} \nu}^{\kappa} h_{k \bar{\mu}}\right) \xi^{\alpha_{\nu}} \bar{\xi}^{\beta \bar{\mu}}$ is semipositive. We note that $(f, g)_{\varphi}=i^{n}(-1)^{n(n-1) / 2} \int_{X}^{\alpha_{0} e^{-\varphi}} e^{-\varphi}(f)$ $\wedge \bar{g}$ if $f, g \in L_{\varphi}^{n, 0}(X, E)$. In particular $L_{\varphi}^{n, 0}(X, E)$ does not depend on the choice of $d s^{2}$. We say $X$ is a weakly 1 -complete manifold if there exists a $C^{\infty}$ plurisubharmonic function $\varphi: X \rightarrow \mathbb{R}$ such that $X_{c}:=\{x \in X ; \varphi(x)<c\}$ is relatively compact for any $c \in \mathbb{R}$. For the basic materials on weakly 1-complete manifolds, see [O-1]. 
Lemma 1. Let $D \subset X$ be a strongly pseudoconvex domain with $C^{\infty}$ smooth boundary, let $\varphi_{0}$ be a $C^{\infty}$ defining function of $D$, and let $\psi$ be a nonnegative $C^{\infty}$ function defined on $\bar{D}$. Then, for any $\varepsilon>0$ and a compact subset $K \subset D$, there exists a $C^{\infty}$ function $\psi_{K}$ on $D$ satisfying the following properties.

(i) $\psi \geqq \psi_{K}$ and $\psi-\psi_{K}=\varepsilon$ on $K$.

(ii) $\inf _{D} \psi_{K}=0$ and $D^{c}:=\left\{x \in D ; \psi_{K}(x) \geqq c\right\}$ is compact for all $c \in(-\infty, 0)$.

(iii) $\left|d \psi_{K} \wedge d \varphi_{0}\right| \leqq C$, where $C$ does not depend on the choice of $K$.

(iv) $\psi-\psi_{K}$ is plurisubharmonic.

Proof. Since $D$ is strongly pseudoconvex, we may assume that $\varphi_{0}$ is strictly plurisubharmonic on a neighbourhood of $\partial D$. Let $\delta$ be any positive number satisfying $\delta<-\sup _{\boldsymbol{K}} \varphi_{0}$ and let $\lambda_{\delta}$ be a $C^{\infty}$ function on $(-\infty, 0)$ such that $\lambda_{\delta}(t), \lambda_{\delta}^{\prime}(t), \lambda_{\delta}^{\prime \prime}(t) \geqq 0$ for all $t, \lambda_{\delta}^{\prime}(t)=-t^{-1}$ on $(-\delta / 2,0)$, and $\lambda_{\delta}(t)=0$ on $(-\infty$, $-\delta)$. Then, for any $\varepsilon>0$ and $\tau>0$ there exists a $\delta_{0}>0$ such that the function

$$
\Phi_{\delta, \varepsilon}^{\tau}:=\tau \lambda_{\delta}\left(\varphi_{0}\right)-\psi-\varepsilon
$$

satisfies

$$
\partial \bar{\partial} \Phi_{\delta, \varepsilon}^{\tau} \geqq \frac{-\tau}{2 \varphi_{0}} \partial \bar{\partial} \varphi_{0}+\frac{1}{4 \tau} \partial \Phi_{\delta, \varepsilon}^{\tau} \bar{\partial} \Phi_{\delta, \varepsilon}^{\tau} \quad \text { on } \quad D \backslash D_{-\delta / 2}
$$

if $0<\delta<\delta_{0}$.

Note that $\Phi_{\delta, \varepsilon}^{\tau}<-\varepsilon / 2$ on $D_{-\delta / 2}$ if $\tau<-\frac{\varepsilon}{2}(\log 2)^{-1}$. Let $\chi: R \rightarrow R$ be a $C^{\infty}$ increasing function such that $\chi(t)=t$ on $(-\infty,-\varepsilon / 2)$ and $\chi(t)=-t^{-1}$ on $(2, \infty)$. If we put $\psi_{K}=-\chi\left(\Phi_{\delta, \varepsilon}^{\tau}\right)$ for $\delta \ll \tau<-\frac{\varepsilon}{2}(\log 2)^{-1}$, then $\psi_{K}$ satisfies (i) through (iv). In fact, (i), (ii), (iii) are trivial and (iv) follows from (*).

Proposition 2. Let $D \subset X$ be a strongly pseudoconvex domain with $C^{\infty}$ boundary, and let $\psi$ be a nonnegative $C^{\infty}$ function defined on $\bar{D}$. Then, for any $C^{\infty}$ function $\varphi$ on $\bar{D}$ and $a C^{\infty}$ E-valued $(n, q)$-form $u$ on $\bar{D}$ with $\left.\overline{* u}\right|_{\partial D}=0$,

$$
\begin{aligned}
& \left\|\sqrt{\psi} \bar{\partial}_{\varphi}^{*} u\right\|_{\varphi, D}^{2}+\|\sqrt{\psi} \bar{\partial} u\|_{\varphi, D}^{2} \\
& \quad \geqq(i e(\psi(\partial \bar{\partial} \varphi+\Theta)-\partial \bar{\partial} \psi) \Lambda u, u)_{\varphi, D}+2 \operatorname{Re}\left(e(\bar{\partial} \psi) \bar{\partial}_{\varphi}^{*} u, u\right)_{\varphi, D},
\end{aligned}
$$

where * denotes the Hodge's star operator,

$$
\left\|\sqrt{\psi} \bar{\partial}_{\varphi}^{*} u\right\|_{\varphi, D}^{2}=\int_{D} \psi e^{-\varphi}\left|\bar{\partial}_{\varphi}^{*} u\right|^{2} d V_{X}, \text { etc. }
$$

Proof. Let $K \subset D$ be any compact subset and let $\psi_{K}$ be chosen for $\psi$ as in Lemma 1. Then, for any $u$ as above, 
(**)

$$
\begin{aligned}
& \left\|\sqrt{\psi_{K}} \bar{\partial}_{\varphi}^{*} u\right\|_{\varphi, D}^{2}+\left\|\sqrt{\psi_{K}} \bar{\partial} u\right\|_{\varphi, D}^{2} \\
& \quad \geqq\left(i e\left(\psi_{K}(\partial \bar{\partial} \varphi+\Theta)-\partial \bar{\partial} \psi_{K}\right) \Lambda u, u\right)_{\varphi, D} \\
& \quad+\left(e\left(\bar{\partial} \psi_{K}\right) \bar{\partial}_{\varphi}^{*} u, u\right)_{\varphi, D}+\left(\bar{\partial} e\left(\bar{\partial} \psi_{K}\right)^{*} u, u\right)_{\varphi, D}
\end{aligned}
$$

(cf. [O-2] $§ 1,(6)$ ).

Since $\left.\overline{* u}\right|_{\partial D}=0$,

$$
\left(\bar{\partial} e\left(\bar{\partial} \psi_{K}\right)^{*} u, u\right)_{\varphi, D}=\left(u, e\left(\bar{\partial} \psi_{K}\right) \bar{\partial}_{\varphi}^{*} u\right)_{\varphi, D} .
$$

By (i) and (iii),

$$
\left\|e\left(\bar{\partial} \psi_{K}\right) * u-e(\bar{\partial} \psi)^{*} u\right\|_{\varphi, D} \leqq \text { const. }\|u\|_{\varphi, D \backslash K} .
$$

By (iv),

$$
\begin{gathered}
\left(i e\left(\psi_{K}(\partial \bar{\partial} \varphi+\Theta)-\partial \bar{\partial} \psi_{K}\right) \Lambda u, u\right)_{\varphi, D} \\
\geqq(i e(\psi \partial \bar{\partial} \varphi-\partial \bar{\partial} \psi) \Lambda u, u)_{\varphi, D} .
\end{gathered}
$$

Thus, taking the limit of the inequality (**) we obtain the desired estimate.

In order to apply the estimate (2) effectively we have to digress a bit into linear algebra.

Let $V$ be a complex vector space of dimension $n$ and let $s_{1}$ be a Hermitian form on $V$. Let $V^{*} \otimes \mathbb{C}=V_{+}^{*} \oplus V^{*}$ be the decomposition into the $\pm \sqrt{-1}$ eigenspaces of the complex structure and let $V_{s_{1}}^{*, q}$ be the subspace of $\left(\bigwedge^{n} V_{+}^{*}\right) \otimes$ $\left(\bigwedge^{q} V_{-}^{*}\right) \subset \bigwedge^{n+q}\left(V^{*} \otimes C\right)$ spanned by the vectors $u \wedge\left(\bar{u}_{1} \wedge \cdots \wedge \bar{u}_{q}\right)$, where $u \in \bigwedge^{n} V_{+}^{*}$ and $u_{k}(\xi)=0$ for $1 \leqq k \leqq q$ on $\left\{\xi \in V \otimes \mathbb{C} ; s_{1}(\xi, \xi)=0\right\}$. Let $\left\{v_{1}, \cdots, v_{n}\right\}$ be a basis of $V_{+}^{*}$ such that $s_{1}=\sum_{\alpha=1}^{l} v_{\alpha} \otimes \bar{v}_{\alpha}-\sum_{\beta=l+1}^{m} v_{\beta} \otimes \bar{v}_{\beta}$. Then $V_{s_{1}}^{*, q}$ is spanned by $u \wedge\left(\overline{v_{i_{1}}} \wedge \cdots \wedge \overline{v_{i_{q}}}\right)$, where $u \in \wedge^{n} V_{+}^{*}$ and $1 \leq i_{1}<\cdots<i_{q} \leq m$. The star operator

$$
*_{s_{1}}: V_{s_{1}}^{*, q} \rightarrow \bigwedge^{n-q}\left(V^{*} \otimes C\right)
$$

is defined as a uniquely determined linear map which satisfy

$$
\begin{aligned}
& *_{s_{1}}\left(v_{1} \wedge \cdots \wedge v_{n} \wedge \overline{v_{j_{1}}} \wedge \cdots \wedge \overline{v_{j_{q}}}\right) \\
& \quad=v_{j_{q+1}} \wedge \cdots \wedge v_{j_{n}} \circ \operatorname{sgn}\left[\begin{array}{l}
1 \cdots n \\
j_{1} \cdots j_{n}
\end{array}\right]\left(\frac{-i}{2}\right)^{n}(-n)^{n(n-1) / 2} \\
& \quad \times \varepsilon_{j_{1}} \cdots \varepsilon_{j_{q}} \cdot
\end{aligned}
$$

Here $\varepsilon_{j}=1$ if $1 \leq j \leq l, \varepsilon_{j}=-1$ if $l+1 \leq j \leq m$ and $\varepsilon_{j}=0$ if $m<j \leq n$.

Then we have a nondegenerate pairing 


$$
\begin{gathered}
V_{s_{1}}^{*, q} \times V_{s_{1}}^{*, q} \rightarrow \bigwedge^{2 n}\left(V^{*} \otimes \boldsymbol{C}\right) \\
\Psi \\
(u, v) \mapsto u \wedge \overline{*_{s_{1}} v}
\end{gathered}
$$

Let $\omega_{s_{1}}$ be the imaginary part of $s_{1}$ and denote by $e\left(\omega_{s_{1}}\right)$ the multiplication by $\omega_{s_{1}}$. Let $s_{2}$ be any positive Hermitian form on $V$. We denote by $e\left(\omega_{s_{1}}\right) *$ the adjoint of $e\left(\omega_{s_{1}}\right)$ with respect to $s_{2}$. We put

$$
\begin{aligned}
& \langle u, v\rangle_{s_{2}} \frac{\omega_{s_{2}}^{n}}{n !}=u \wedge \overline{*_{s_{2}} v} \\
& \langle u, v\rangle_{s_{1}} \frac{\omega_{s_{2}}^{n}}{n !}=u \wedge \overline{*_{s_{1}} v} \quad\left(v \in V_{s_{1}}^{*, q}\right) .
\end{aligned}
$$

Then we have

$$
\langle v, v\rangle_{s_{1}}=\left\langle e\left(\omega_{s_{2}}\right) e\left(\omega_{s_{1}}\right)^{-1} v, v\right\rangle_{s_{2}} \text { for } v \in V_{s_{1}}^{*, 1} .
$$

Here $e\left(\omega_{s_{1}}\right)^{-1}$ denotes the inverse map of $e\left(\omega_{s_{1}}\right): \bigwedge^{n-1} V_{+}^{*} \rightarrow V_{s_{1}}^{*, 1}$. If $s_{1}$ is semipositive, then

$$
\left|\langle u, v\rangle_{s_{2}}\right|^{2} \leq\left\langle e\left(\omega_{s_{1}}\right) e\left(\omega_{s_{2}}\right)^{*} u, u\right\rangle_{s_{2}}\langle v, v\rangle_{s_{1}}
$$

for any $u \in \bigwedge^{n+1}\left(V^{*} \otimes C\right)$ and $v \in V_{s_{1}}^{*, 1}$.

Let $\left(W, h_{1}\right)$ be another Hermitian vector space. Then the inner product $\langle v, v\rangle_{s_{1}}$ is naturally extended to $W \otimes V_{s_{1}}^{*, 1}$, which will be also denoted by $\langle v, v\rangle_{s_{1}}$. We have similar estimates as (3) for the elements of $W \otimes\left(\bigwedge^{n+1} V^{*} \otimes C\right)$ and $W \otimes V_{s_{1}}^{*, 1}$.

Thus we have the following inequality for the bundle valued forms.

Proposition 3. Let $\sigma$ be a semipositive $(1,1)$-form on $X$ and let $u, v \in$ $L_{\varphi}^{n, 1}(X, E)$. If $v(x) \in E_{x} \otimes\left(T_{X, x}\right)_{\sigma(x)}^{*}, 1$ for any $x \in X$, then

$$
\left|(u, v)_{\varphi}\right|^{2} \leq(e(\sigma) \Lambda u, u)_{\varphi} \int_{X} e^{-\varphi}\langle v, v\rangle_{\sigma} \quad d V_{X}
$$

and

$$
\int_{X} e^{-\varphi}\langle v, v\rangle_{\sigma} d V_{X}=\left(e(\omega) e(\sigma)^{-1} v, v\right)_{\varphi}
$$

To simplify the notation we set

$$
\begin{aligned}
& L_{\varphi}^{n, q}(X, E)_{\sigma} \\
& \quad=\left\{v \in L_{\varphi}^{n, q}(X, E) ; v(x) \in\left(T_{X, x}\right)_{\sigma}^{*, q} \text { for any } x \in X\right\} .
\end{aligned}
$$

Proposition 4. Let $D \subset X$ be a strongly pseudoconvex domain with $C^{\infty}$-smooth 
boundary, $\psi$ a nonnegative $C^{\infty}$ function on $\bar{D}$, and $\varphi$ a $C^{\infty}$ function on $\bar{D}$. Suppose that $(E, h)$ is Nakano semipositive and there exists a positive locally bounded function $\eta$ on $D$ such that

$$
\sigma(\eta):=\psi \partial \bar{\partial} \varphi-\partial \bar{\partial} \psi-\eta^{2}|\partial \psi|^{-2} \partial \psi \bar{\partial} \psi
$$

is semipositive. Then, for any $C^{\infty}$ E-valued $(n, 1)$-form $u$ on $\bar{D}$ with $\left.{ }^{*} u\right|_{\partial D}=0$ and $v \in L_{\varphi}^{n, 1}(D, E)_{\sigma(\eta)}$,

$$
\begin{aligned}
& \left|(u, v)_{\varphi, D}\right|^{2} \leq\left(e(\omega) e(\sigma(\eta))^{-1} v, v\right)_{\varphi, D} \\
& \quad \times\left(\left\|(\sqrt{\psi}+\eta|\partial \psi|) \partial_{\varphi}^{*} u\right\|_{\varphi, D}^{2}+\|\sqrt{\psi} \bar{\partial} u\|_{\varphi, D}^{n}\right) .
\end{aligned}
$$

\section{§2. Proof of Theorem}

Let the notations be as in the introduction. Since $X$ is a Stein manifold one can find a decreasing sequence of $C^{\infty}$ plurisubharmonic functions $\left\{\varphi_{\mu}\right\}_{\mu=1}^{\infty}$ which converges to $\varphi$ almost everywhere. Hence it suffices to prove Theorem in case $\varphi$ is $C^{\infty}$. Moreover we may assume that $d s_{1} \wedge \cdots \wedge d s_{m} \neq 0$ everywhere. In fact, take an analytic subset $Z \subset X$ of codimension one such that

$$
Z \supset\left\{x ;\left.d s_{1} \wedge \cdots \wedge d s_{m}\right|_{x}=0\right\} \text {. }
$$

Then it suffices to show the extendability of $g \wedge d s_{1} \wedge \cdots \wedge d s_{m}$ to $X \backslash Z$, since the apparent singularity along $Z$ is improper in virtue of the $L^{2}$ condition.

As in [O-T] we fix an increasing family of strongly pseudoconvex domains $X_{1} \subset X_{2} \Subset \ldots \Subset X_{\mu} \subset \ldots$ with $C^{\infty}$ smooth boundaries such that

$$
X=\bigcup_{\mu=1}^{\infty} X_{\mu}
$$

Then it suffices to find the extensions to $X_{\mu}$, since one obtains a desired extension as a weak limit of a subsequence of the extensions to $X_{\mu}(\mu \rightarrow \infty)$.

Let $G$ be an arbitrary holomorphic extension of $g \wedge d s_{1} \wedge \cdots \wedge d s_{m}$ to $X$. It certainly exists since $X$ is a Stein manifold. Let $\chi: \mathbb{R} \rightarrow \mathbb{R}$ be a $C^{\infty}$ function satisfying $\chi(t)=1$ on $(-\infty, 1 / 2)$ and $\chi(t)=0$ on $(1, \infty)$. For any $\delta>0$ we put

$$
G^{[\delta]}=\left\{\begin{array}{l}
\chi\left(|s|^{2} / \delta^{2}\right) G \\
\text { on }\{x \in X ;|s(x)|<\delta\} \\
0 \text { otherwise. }
\end{array}\right.
$$

Then $G^{[\delta]}$ is a $C^{\infty}$ extension of $g \wedge d s_{1} \wedge \cdots \wedge d s_{m}$. We put

$$
v^{\delta}=\bar{\partial} G^{[\delta]} \text {. }
$$


Note that $v^{\delta}=0$ on a neighbourhood of $Y$. Taking an arbitrary Kähler metric $d s_{0}^{2}$ of $X$, we fix a metric $d s^{2}$ of $X$ by

$$
d s^{2}=d s_{0}^{2}+2 \partial \bar{\partial} \log \left(1+|s|^{2}\right) .
$$

Then, for any $\mu$ one can find a sufficiently small $\delta_{\mu}$ such that

$$
\begin{aligned}
& \int_{X_{\mu}} e^{-\varphi}|s|^{-2 m} \delta^{2}\left|v^{\delta}\right|^{2} d V_{X} \\
& \quad \leq C_{m} \int_{Y} e^{-\varphi}|g|^{2} d V_{Y} \quad \text { if } \quad \delta<\delta_{\mu} .
\end{aligned}
$$

Here $C_{m}$ depends only on $m$. Let $\lambda: \boldsymbol{R} \rightarrow \boldsymbol{R}$ be a $C^{\infty}$ function satisfying $\lambda^{\prime}(t) \geq$ $0, \lambda^{\prime \prime}(t) \geq 0, \sup \lambda^{\prime}(t) \leq 1, \sup \lambda^{\prime \prime}(t)<2 / 3$, and

$$
\lambda(t)=\left\{\begin{array}{ccl}
0 & \text { on } & (-\infty, 0) \\
t-1 & \text { on } & (2, \infty) .
\end{array}\right.
$$

We put $\psi_{0}=\lambda\left(-\log \left(|s|^{2}+\delta^{2}\right)\right)$. Then $\partial \bar{\partial} \psi_{0}=-\partial \bar{\partial} \log \left(|s|^{2}+\delta^{2}\right)$ if $|s|^{2}+\delta^{2}<$ $e^{-2}, \partial \bar{\partial} \psi_{0}=0$ if $|s|^{2}+\delta^{2}>1$, and

$$
\partial \bar{\partial} \psi_{0} \leq \frac{8 e^{4}}{3} \partial \bar{\partial} \log \left(|s|^{2}+1\right)
$$

if $e^{-2} \leq|s|^{2}+\delta^{2} \leq 1$.

On the other hand

$$
\partial \psi_{0} \bar{\partial} \psi_{0} \leq\left(|s|^{2}+1\right)^{2}\left(|s|^{2}+\delta^{2}\right)^{-1} \partial \bar{\partial} \log \left(|s|^{2}+1\right) .
$$

Thus $\left|\partial \psi_{0}\right|^{2}$ is estimated from above by $\left(|s|^{2}+1\right)^{2}\left(|s|^{2}+\delta^{2}\right)^{-1}$. For any $\varepsilon>0$ we put

$$
\psi_{\varepsilon}=\psi_{0}+\varepsilon^{-1}\left(\frac{8 e^{4}}{3}+4\right)
$$

Then $\left|\partial \psi_{\varepsilon}\right|^{2} \leq\left(|s|^{2}+1\right)^{2}\left(|s|^{2}+\delta^{2}\right)^{-1}$ and

$$
\psi_{\mathrm{g}} \leq \alpha_{\mathrm{g}}\left(|s|^{2}+\delta^{2}\right)^{-1}
$$

where

$$
\alpha_{\varepsilon}=\sup _{|s|<1}\left(|s|^{2}+\delta^{2}\right)\left(-\log \left(|s|^{2}+\delta^{2}\right)+\varepsilon^{-1}\left(\frac{8 e^{4}}{3}+4\right)\right)
$$

We put

$$
\varphi_{\mathrm{\varepsilon}}=\varphi+2 m \log |s|+\varepsilon \log \left(|s|^{2}+1\right)
$$

Then 


$$
\sigma_{\varepsilon}:=\psi_{\varepsilon} \partial \bar{\partial} \varphi_{\varepsilon}-\partial \bar{\partial} \psi_{\varepsilon}-\left|\partial \psi_{\varepsilon}\right|^{-2} \partial \psi_{\varepsilon} \bar{\partial} \psi_{\varepsilon}
$$

is semipositive and

$$
\sigma_{\varepsilon} \geq \partial \bar{\partial} \log \left(|s|^{2}+\delta^{2}\right)
$$

on $\{x ;|s(x)|<\delta\}$.

Combining it with (4) we see that there exists a $\delta_{\mu}^{\prime}>0$ such that

$$
\begin{aligned}
& \left(e(\omega) e\left(\sigma_{\varepsilon}\right)^{-1} v^{\delta}, v^{\delta}\right)_{\varphi_{\varepsilon}, X_{\mu}} \\
& \quad \leq 4 C_{m} \int_{Y} e^{-\varphi_{\varepsilon}}|g|^{2} d V_{Y} \text { if } \delta<\delta_{\mu}^{\prime} .
\end{aligned}
$$

Therefore by Proposition $\psi$, if $\delta<\delta_{\mu}^{\prime}$

$$
\begin{aligned}
& \left|\left(u, v^{\delta}\right)_{\varphi_{\varepsilon}, X_{\mu}}\right|^{2} \\
& \quad \leq 4 C_{m} \int_{Y} e^{-\varphi_{\varepsilon}}|g|^{2} d V_{Y} \\
& \quad \times\left\{\left\|\left(\sqrt{\psi_{\varepsilon}}+\left|\partial \psi_{\varepsilon}\right|\right) \bar{\partial}_{\varphi_{\varepsilon}}^{*} u\right\|_{\varphi_{\varepsilon}, X \mu}^{2}+\left\|\sqrt{\psi_{\varepsilon}} \bar{\partial} u\right\|_{\varphi_{\varepsilon}, X \mu}^{2}\right\}
\end{aligned}
$$

for any $C^{\infty} E$-valued $(n, 1)$-form $u$ on $\bar{X}_{\mu}$ with $\left.{ }^{*} u\right|_{\partial x_{\mu}}=0$. Since the same estimate also holds for $u \in \operatorname{Dom} \bar{\partial}_{\varphi_{\varepsilon}}^{*} \cap \operatorname{Dom} \bar{\partial} \cap L_{\varphi_{\varepsilon}}^{n, 1}\left(X_{\mu}, E\right)$, there exists a solution $b_{\mathrm{q}}^{\delta}$ to the equation $\bar{\partial}\left(\left(\sqrt{\psi_{\mathrm{q}}}+\left|\partial \psi_{\mathrm{\varepsilon}}\right|\right) b_{\varepsilon}^{\delta}\right)=v^{\delta}$ with

$$
\left\|b_{\varepsilon}^{\delta}\right\|_{\varphi_{\varepsilon}, X_{\mu}}^{2} \leq 4 C_{m} \int_{Y} e^{-\varphi_{\varepsilon}}|g|^{2} d V_{Y}
$$

(cf. $[\mathrm{H}])$.

We put

$$
G_{\varepsilon}:=G^{[\delta]}-\left(\sqrt{\psi_{\varepsilon}}+\left|\partial \psi_{\varepsilon}\right|\right) b_{\varepsilon}^{\delta} .
$$

Then $G_{\varepsilon}$ is a holomorphic extension of $g \wedge d s_{1} \wedge \cdots \wedge d s_{m}$ to $X_{\mu}$. The verification of the $L^{2}$ estimate is left to the reader.

Proof of Corollary 1. Let $\varphi: X \rightarrow \mathbb{R}$ be any $C^{\infty}$ plurisubharmonic exhaustion function and let $(B, a)$ be a positive line bundle over $X$. Then, for any $c \in \mathbb{R}, X_{c}:=\{x ; \varphi(x)<c\}$ is embeddable into a projective space by holomorphic sections of $B^{m}(m=m(c) \gg 0)$. In particular there exists a proper analytic subset $Z_{c} \subset X_{c}$ such that $Z_{c} \nsupseteq Y$ and $X_{c} \backslash Z_{c}$ is a Stein manifold. Let $g \wedge d s \in \Gamma\left(Y, \mathcal{O}_{Y}\right.$ $\left.\left(K_{X} \otimes E\right)\right)$ and choose a convex increasing $C^{\infty}$ function $\lambda$ such that

$$
g \in L_{\lambda(\varphi)}^{n-1,0}(Y, E) .
$$

Applying Theorem to the manifolds $X_{c} \backslash Z_{c} \supset Y \cap X_{c} \backslash Z_{c}$ and $g \mid Y \cap X_{c} \backslash Z_{c}$, we 
have extensions of $g \wedge d s$ to $X_{c} \backslash Z_{c}$ whose norms in $L_{\lambda(\varphi)}^{n, 0}\left(X_{c} \backslash Z_{c}, E\right)$ are dominated by const. $\|g\|_{\lambda(\varphi)}$. Since the singularities along $Z_{c}$ are improper, by taking a weak limit of these extended forms in $L_{\lambda(\varphi)}^{n, 0}(X, E)$ we obtain a holomorphic extension of $g \wedge d s$ to $X$.

Remark 1. The assumption that $X$ admits a positive line bundle was only used to ensure the existence of the divisors $Z_{c}$. Hence one can replace the existence of a positive bundle in the hypothesis by the existence of a Zariski dense Stein open subset $\Omega \subset X$ such that $X \backslash \Omega$ does not contain any connected component of $Y$.

Remark 2. Corollary 1 may be regarded as an extension of KazamaNakano's vanishing theorem on weakly 1-complete manifolds (cf. [O-1]). In fact, if $E$ is Nakano-positive then $H^{1}\left(X, \mathcal{O}\left(K_{X} \otimes E\right)\right)=0$ so that the surjectivity of the above map follows immediately. H. Skoda [S-2] has established a similar surjectivity theorem on weakly 1 -complete manifolds as a generalization of his $L^{2}$ corona theorem on pseudoconvex domains in $\mathbb{C}^{n}$ (cf. [S-1]).

Proof of Corollary 2. Let $w_{1}, \cdots, w_{k}$ be holomorphic functions on $\boldsymbol{C}^{N}$ which generate the stalks of the ideal sheaf of $Y$ at each point of $\bar{\Omega}$. Let $m=\operatorname{codim} Y$. Then for each $m$-tuple $\left(w_{i_{1}}, \cdots, w_{i m}\right)$ we apply Theorem as follows. Let $\Sigma_{I} \subset$ $C^{N}\left(I=\left(i_{1}, \cdots, i_{m}\right)\right)$ be an analytic subset of codimension one which contains the set $\left\{x \in Y ; d w_{i_{1}} \wedge \cdots \wedge d w_{i_{n}}(x)=0\right\}$, and let $\sigma_{I}$ be a defining function of $\Sigma_{I}$. Then, by Rückert's theorem there exists a $p \in \mathbb{N}$ such that for all $I$

$$
\sigma_{I}^{p} d z_{1} \wedge \cdots \wedge d z_{N}=g_{I} \wedge d w_{i_{1}} \wedge \cdots \wedge d w_{i_{m}} \text { on } \quad Y
$$

for some holomorphic $(N-m)$-form $g_{I}$ on $Y$. Here $\left(z_{1}, \cdots, z_{N}\right)$ denotes the coordinate of $C^{N}$. Then $f \sigma_{I}^{p} d z_{1} \wedge \cdots \wedge d z_{N}$ has an extension $G_{I}$ to $\mathcal{Q}$ with

$$
\left|\int_{\Omega} e^{-\varphi} G_{I} \wedge \bar{G}_{I}\right| \leq\left. C_{I}\left|\int_{Y \cap \Omega} e^{-\varphi}\right| f\right|^{2} g_{I} \wedge \bar{g}_{I} \mid
$$

where $C_{I}$ does not depend on $f$. Let $\eta_{I}$ be holomorphic functions on $\mathbb{C}^{N}$ satisfying

$$
\sum_{\substack{I \subset\{1, \cdots, k\} \\ \sharp I=m}} \sigma_{I}^{p} \eta_{I}=1 \quad \text { on } \quad Y
$$

Then we define a function $F$ by

$$
F d z_{1} \wedge \cdots \wedge d z_{n}=\sum_{\substack{I \subset\{1, \cdots, k\} \\ \forall I=m}} \eta_{I} G_{I} .
$$


Clearly $F$ is an extension of $f$ with desired properties.

Remark. Corollary 2 is easily generalized to relatively compact pseudoconvex domains of Stein manifolds. The detail is left to the reader.

\section{References}

[H] Hörmander, L., $L^{2}$ estimate and existence theorems for the $\bar{\partial}$ operator, Acta Math. 113 (1965), 89-152.

[O-1] Ohsawa, T., Cohomology vanishing theorenus on weakly 1-complete manifolds, Publ. RIMS, Kyoto Univ. 19 (1983), 1181-1201.

[O-2] — - On the rigidity of noncompact quotients of bounded symmetric domains, to appear in Publ. Math. Kyoto Univ., 23.

[O-T] Ohsawa, T, and Takegoshi, K., On the extension of $L^{2}$ holomorphic functions, Math. Zeit. 195 (1987), 197-204.

[S-1] Skoda, H., Application des techniques $L^{2}$ à la théorie des idéaux d'une algèbre de fonctions holomorphes avec poids, Ann. scient. Ec. Norm. Sup., 5 (1972), 545-579.

[S-2] — Morphismes surjectifs de fibrés vectoriels sémipositifs, Anit. scient. Ec. Norm. Sup., $\mathbb{1} 1$ (1978), 577-611. 
